\title{
Non-Abelian strings in $\mathcal{N}=1$ supersymmetric $Q C D$ *
}

\author{
Evgenii Ievlev ${ }^{1,2, * *}$ and Alexei Yung ${ }^{1,2,3, * * *}$ \\ ${ }^{1}$ National Research Center "Kurchatov Institute”, Petersburg Nuclear Physics Institute, Gatchina, St. \\ Petersburg 188300, Russia \\ ${ }^{2}$ St. Petersburg State University, Universitetskaya nab., St. Petersburg 199034, Russia \\ ${ }^{3}$ William I. Fine Theoretical Physics Institute, University of Minnesota, Minneapolis, MN 55455
}

\begin{abstract}
.
Non-Abelian flux tubes (strings) are well studied in $\mathcal{N}=2$ supersymmetric QCD in $(3+1)$ dimensions. In addition to translational zero modes they have also orientational moduli associated with rotations of their fluxes inside a nonAbelian group. The dynamics of the orientational moduli is described by the two dimensional $\mathrm{CP}(N-1)$ model living on the world sheet of the non-Abelian string. In this paper we consider a deformation of $\mathcal{N}=2$ supersymmetric QCD with the $\mathrm{U}(N)$ gauge group and $N_{f}=N$ quark flavors with a mass term $\mu$ of the adjoint matter. In the limit of large $\mu$ the theory flows to an $\mathcal{N}=1$ supersymmetric QCD. We study the solution for the non-Abelian string in this limit and derive an effective theory on the string world sheet. The bosonic sector of this theory is still given by the $\mathrm{CP}(N-1)$ model. We study also the fermionic sector of the world sheet theory. Upon the deformation the non-Abelian string is no longer BPS and we show that the fermionic superorientational zero modes are all lifted. This leaves us with the pure bosonic $\mathrm{CP}(N-1)$ model on the string world sheet in the limit of $\mathcal{N}=1 \mathrm{QCD}$. We discuss what happens to confined monopoles at large $\mu$.
\end{abstract}

\section{Introduction}

The mechanism of confinement based on a monopole condensation [2] was shown to work $[3,4]$ in the monopole vacua of $\mathcal{N}=2$ supersymmetric QCD. This confinement is essentially Abelian [5-8]. Searchers for non-Abelian generalization of Seiberg-Witten scenario lead to the discovery of non-Abelian strings [9-12], see also [13-16] for a review. These BPS nonAbelian vortices are formed in $\mathcal{N}=2 \mathrm{SU}(N) \times \mathrm{U}(1)$ QCD when the theory is in the Higgs phase for quarks, and monopoles are confined. Apart from the translational zero modes of the usual ANO string, the non-Abelian vortex has also orientational moduli associated with rotations of their fluxes inside the non-Abelian $\mathrm{SU}(N)$ group. Internal dynamics of the orientational moduli is governed by the $\mathrm{CP}(N-1)$ model living on the string world sheet.

\footnotetext{
${ }^{*}$ This talk is based on the paper [1] and some new results.

**e-mail: ievlevea@thd.pnpi.spb.ru

***e-mail: yung@thd.pnpi.spb.ru
} 
In the recent years there has been some progress in studies of non-Abelian vortices in $\mathcal{N}=1$ gauge theories. This can be done by introducing a so-called $\mu$-deformation which is a mass term for the adjoint matter. When $\mu$ goes to infinity, the $\mathcal{N}=2$ QCD flows to $\mathcal{N}=1$ QCD. This setup for $\mathcal{N}=2$ supersymmetric QCD with $\mathrm{U}(N)$ gauge group and $N_{f}=N$ flavors of massless quarks was considered in [17-22]. Quark condensation is triggered by the Fayet-Iliopoulos (FI) $D$-term. It was found that the vortices remain BPS saturated, but the fermionic orientational moduli change. The world-sheet theory becomes heterotic $\mathcal{N}=(0,2)$ $\mathrm{CP}(N-1)$ model.

Here we study a more natural setup. We consider the $\mu$-deformation of $\mathcal{N}=2 \mathrm{QCD}$ without a FI term in a quark vacuum. Squark condensate is determined by $\sqrt{\mu m}$, were $m$ is a quark mass. In the large $\mu$ limit the theory flows to $\mathcal{N}=1 \mathrm{QCD}$ in the quark vacuum. Non-Abelian strings cease to be BPS saturated and both bosonic and fermionic profile of the string are modified. We study solutions for the non-Abelian string profile functions in the large $\mu$ limit and derive the effective theory on the string world sheet.

We show that the fermionic sector of the world-sheet theory decouples, while the bosonic part is still given by the $\mathrm{CP}(N-1)$ model. In the limit of equal quark masses the confined monopoles survive [1] and seen as kinks of the world-sheet theory [11, 12].

We further consider the case with "extra" quark flavors, $N_{f}>N$. In this case non-Abelian "semilocal" strings are formed in $\mathcal{N}=2$ QCD. They have additional $\left(N_{f}-N\right)$ size moduli. We find that after switching on the $\mu$ deformation these size moduli develop a potential. This potential forces the size of a semilocal string to shrink and it transforms into a local string.

\section{Theoretical setup}

We start by describing the field content of the four-dimensional $\mathcal{N}=2$ supersymmetric QCD with the gauge group $\mathrm{SU}(N) \times \mathrm{U}(1)$. The matter consists of $N_{f}=N+\tilde{N}$ flavors of quark hypermultiplets in the fundamental representation with squarks $q^{k A}$ and $\widetilde{q}_{A k}$ two-component Weyl fermions $\psi^{k A}$ and $\widetilde{\psi}_{A k}$. Here, $A=1, \ldots, N_{f}$ is the flavor index and $k=1, \ldots, N$ is the color index. We use the same notation for the quark superfields and their scalar components. The vector multiplets consist of $\mathrm{U}(1)$ gauge field $A_{\mu}$ and $\mathrm{SU}(N)$ gauge field $A_{\mu}^{a}$, complex scalar fields $a$ and $a^{a}$ in the adjoint representation of the color group, and their Weyl fermion superpartners $\left(\lambda_{\alpha}^{1}, \lambda_{\alpha}^{2}\right)$ and $\left(\lambda_{\alpha}^{1 a}, \lambda_{\alpha}^{2 a}\right)$. Index $a$ runs from 1 to $N^{2}-1$, and the spinorial index $\alpha=1,2$.

Superpotential of the $\mathcal{N}=2$ SQCD is

$$
\mathcal{W}_{\mathcal{N}=2}=\sqrt{2}\left\{\widetilde{q}_{A} \mathcal{A}^{\mathrm{U}(1)} q^{A}+\widetilde{q}_{A} \mathcal{A}^{a} T^{a} q^{A}\right\}+m_{A} \widetilde{q}_{A} q^{A},
$$

The $\mu$ deformation considered in this paper is given by the superpotential

$$
W_{\mathcal{N}=1}=\sqrt{2 N} \mu_{1}\left(\mathcal{A}^{\mathrm{U}(1)}\right)^{2}+\frac{\mu_{2}}{2}\left(\mathcal{A}^{a}\right)^{2} .
$$

It breaks $\mathcal{N}=2$ supersymmetry down to $\mathcal{N}=1$.

In the special case when

$$
\mu \equiv \mu_{2}=\mu_{1} \sqrt{\frac{2}{N}},
$$

superpotential (2.2) becomes a single trace operator

$$
\mathcal{W}_{\mathcal{N}=1}=\mu \operatorname{Tr}\left(\Phi^{2}\right)
$$


where we defined a scalar adjoint matrix as

$$
\Phi=\frac{1}{2} a^{U(1)}+T^{a} a^{a} .
$$

We will consider bulk QCD in the limit of large $\mu_{1}$ and $\mu_{2}$, when the adjoint matter decouples and the theory becomes $\mathcal{N}=1 \mathrm{QCD}$. Integrating out the adjoint matter in a sum of superpotentials (2.1) and (2.2) we get a quark superpotential of our $\mu$-deformed bulk theory

$$
\mathcal{W}(q, \tilde{q})=-\frac{1}{2 \mu_{2}}\left[\left(\tilde{q}_{A} q^{B}\right)\left(\tilde{q}_{B} q^{A}\right)-\frac{\alpha}{N}\left(\tilde{q}_{A} q^{A}\right)^{2}\right]+m\left(\tilde{q}_{A} q^{A}\right)
$$

where we assume for simplicity that quarks have equal masses $m$ and

$$
\alpha=1-\sqrt{\frac{N}{2}} \frac{\mu_{2}}{\mu_{1}} .
$$

In the case of single trace deformation (2.3) $\alpha=0$.

The bosonic action of the theory is

$$
\begin{array}{r}
S_{\text {bos }}=\int d^{4} x\left(\frac{1}{2 g_{2}^{2}} \operatorname{Tr}\left(F_{\mu \nu}^{\mathrm{SU}(N)}\right)^{2}+\frac{1}{4 g_{1}^{2}}\left(F_{\mu \nu}^{\mathrm{U}(1)}\right)^{2}+\right. \\
\left.\left|\nabla_{\mu} q^{A}\right|^{2}+\left|\nabla_{\mu} \overline{\bar{q}}^{A}\right|^{2}+V\left(q^{A}, \widetilde{q}_{A}\right)\right) .
\end{array}
$$

Here $\nabla_{\mu}$ is a covariant derivative

$$
\nabla_{\mu}=\partial_{\mu}-\frac{i}{2} A_{\mu}^{\mathrm{U}(1)}-i A_{\mu}^{a} T^{a},
$$

while the scalar potential $V\left(q^{A}, \widetilde{q}_{A}\right)$ is a sum of the $D$-term and $F$-term potentials,

$$
V\left(q^{A}, \widetilde{q}_{A}\right)=V_{D}\left(q^{A}, \widetilde{q}_{A}\right)+V_{F}\left(q^{A}, \widetilde{q}_{A}\right) .
$$

The $D$-term potential reads

$$
V_{D}=\frac{g_{2}^{2}}{2}\left(\bar{q}_{A} T^{a} q^{A}-\tilde{q}_{A} T^{a} \overline{\tilde{q}}^{A}\right)^{2}+\frac{g_{1}^{2}}{8}\left(\left|q^{A}\right|^{2}-\left|\tilde{q}^{A}\right|^{2}\right)^{2},
$$

while the $F$ term potential is determined by the superpotential (2.6).

In this paper we will consider the vacuum where the maximal possible number of quark flavors equal to $N$ condense (the so called $r=N$ vacuum, where $r$ is the number of condensed squark flavors at weak coupling, see [24] for a review). In this vacuum squark VEVs are given by

$$
\left\langle q^{k A}\right\rangle=\left\langle\overline{\bar{q}}^{k A}\right\rangle=\sqrt{\frac{\xi}{2}}\left(\begin{array}{ccc}
1 & 0 & \ldots \\
\ldots & \ldots & \ldots \\
\ldots & 0 & 1
\end{array}\right)
$$

where we wrote down the squark field as an $N \times N$ matrix in color and flavor indices, and the parameter $\xi$ is defined as

$$
\xi=2 \mu m
$$

for the single trace deformation (2.3). 
To get an $\mathcal{N}=1$ theory we need to sent $\mu \rightarrow \infty$. Note however that if the quark masses remain fixed, the parameters $\xi \sim \mu m$ also go to infinity, and our quark vacuum becomes a run-away vacuum (all the $r$ vacua with the non-zero $r$ become run-away vacua). In this case $\mathcal{N}=1 \mathrm{QCD}$ is a theory with only $N$ vacua which originate from $N$ monopole vacua $(r=0$ vacua) of $\mathcal{N}=2 \mathrm{QCD}$.

Here we define $\mathcal{N}=1$ QCD in a different way, namely, together with the limit $\mu \rightarrow \infty$ we simultaneously make the quark masses small so that the product $\mu m$ (and the quark VEVs) are fixed,

$$
\mu \rightarrow \infty, \quad m \rightarrow 0, \quad \mu m=\text { fixed. }
$$

This way we keep track of all the $r$ vacua present in $\mathcal{N}=2$ QCD. In this paper we will study non-Abelian strings particularly in the $r=N$ quark vacuum (2.12) assuming the limit of large $\mu$ when the bulk theory flows to the generalized $\mathcal{N}=1$ QCD defined above.

So as to keep our bulk theory at weak coupling we assume that the squark VEVs are large as compared with the scale $\Lambda_{\mathcal{N}=1}$ of the $\mathrm{SU}(N)$ sector of $\mathcal{N}=1$ QCD. Namely, we assume that

$$
\sqrt{\mu m} \gg \Lambda_{\mathcal{N}=1} .
$$

Due to the squark condensation (2.12) both (local) gauge and (global) flavor symmetries get broken. The diagonal global $\mathrm{SU}(N)$ survives, however,

$$
\mathrm{U}(N)_{\text {gauge }} \times \mathrm{SU}(N)_{\text {flavor }} \rightarrow \mathrm{SU}(N)_{C+F} .
$$

A color-flavor locking takes place in the vacuum. This fact leads to an emergence of nonAbelian strings, see [15] for a review.

Let us briefly summarize a perturbative spectrum of our bulk theory in the large $\mu$ limit for the case of equal quark masses, cf. [15]. The $\mathrm{U}(N)$ gauge group is completely Higgsed and the masses of the gauge bosons are

$$
m_{G}^{S U(N)}=g_{2} \sqrt{|\xi|}
$$

for the $\mathrm{SU}(N)$ gauge bosons and

$$
m_{G}^{U(1)}=g_{1} \sqrt{\frac{N}{2}} \sqrt{|\xi|}
$$

for the $\mathrm{U}(1)$ one. Below we also assume that the gauge boson masses are of the same order,

$$
m_{G}^{U(1)} \sim m_{G}^{S U(N)} \equiv m_{G}
$$

Extracting a quark mass matrix from potential (2.10) we find that out of $4 N^{2}$ real degrees of freedom of the $q^{k A}$ and $\overline{\bar{q}}^{k A}$ squarks $N^{2}$ phases are eaten by the Higgs mechanism, $\left(N^{2}-1\right)$ real squarks have mass (2.17), while one real squark has mass (2.18). These squarks are scalar superpartners of the $\mathrm{SU}(N)$ and $\mathrm{U}(1)$ gauge bosons in massive vector $\mathcal{N}=1$ supermultiplets, respectively.

Other $2 N^{2}$ squarks become much lighter in the large $\mu$ limit. The masses of $2\left(N^{2}-1\right)$ of them forming the adjoint representation of the global color-flavor $\mathrm{SU}_{C+F}(N)$ (2.16) are given by

$$
m_{L}^{S U(N)}=\left|\frac{\xi}{\mu_{2}}\right|,
$$


while two real $\mathrm{SU}_{C+F}(N)$ color-flavor singlets have mass

$$
m_{L}^{U(1)}=\sqrt{\frac{N}{2}}\left|\frac{\xi}{\mu_{1}}\right|,
$$

If $\mu_{2}$ and $\mu_{1}$ are of the same order (more exactly, we assume below that $\alpha=$ const, see (2.7)), then

$$
m_{L}^{U(1)} \sim m_{L}^{S U(N)} \equiv m_{L} \sim m \ll m_{G} .
$$

Below we will heavily use this mass hierarchy of the perturbative spectrum.

In particular, in the limit (2.14) $m_{L} \rightarrow 0$, and $2 N^{2}$ squarks become massless. This reflects the presence of the Higgs branch which develops in this limit. The presence of massless scalars developing VEVs makes the string solution ill-defined [25, 26]. Below we use the $\mu$-deformed $\mathcal{N}=2$ QCD at large $\mu$ as an infra-red (IR) regularization of $\mathcal{N}=1 \mathrm{QCD}$. At large but finite $\mu$ the Higgs branch present in $\mathcal{N}=1$ QCD is lifted and the IR divergences are regularized, cf. [27].

\section{Non-Abelian strings}

\subsection{Bosonic sector}

In this section we present the solution for the non-Abelian vortex in the $N_{f}=N_{c}$ case. First consider a stationary string along the $x_{3}$ axis. The solution is derived using the ansatz $[10,11,15]$ :

$$
\begin{aligned}
\sqrt{2} q^{k A}=\sqrt{2} \overline{\bar{q}}^{k A} \equiv \varphi & =\phi_{2}+n \bar{n}\left(\phi_{1}-\phi_{2}\right) \\
& =\frac{1}{N}\left(\phi_{1}+(N-1) \phi_{2}\right)+\left(\phi_{1}-\phi_{2}\right)(n \bar{n}-1 / N), \\
A_{i}^{\mathrm{SU}(N)} & =\varepsilon_{i j} \frac{x^{j}}{r^{2}} f_{W}(r)(n \bar{n}-1 / N), \\
A_{i}^{\mathrm{U}(1)} & =\frac{2}{N} \varepsilon_{i j} \frac{x^{j}}{r^{2}} f(r),
\end{aligned}
$$

where the index $i$ runs over the transverse coordinates 1,2. Here we introduced profile functions $\phi_{1}(r)$ and $\phi_{2}(r)$ for scalar quarks and $f(r)$ and $f_{W}(r)$ for gauge bosons, and $r$ is the polar radius in the $x_{1}, x_{2}$ plane. Orientational modes of the non-Abelian string are parametrized by the complex vector $n^{l}, l=1, \ldots, N$, satisfying

$$
\bar{n}_{l} \cdot n^{l}=1
$$

These modes arise due to a possibility to rotate a particular string solution (say, with $n^{l}=\delta^{l 1}$ ) with respect to the unbroken color-flavor global group $\mathrm{SU}(N)_{C+F}$, see (2.16).

Boundary conditions for the gauge and scalar profile functions are

$$
\begin{aligned}
& \phi_{1}(0)=0, \quad \phi_{2}(0) \neq 0, \quad \phi_{1}(\infty)=\sqrt{\xi}, \quad \phi_{2}(\infty)=\sqrt{\xi}, \\
& f_{W}(0)=1, \quad f(0)=1, \quad f_{W}(\infty)=0, \quad f(\infty)=0 .
\end{aligned}
$$


We solve equations of motion for our theory (2.8) using the above ansatz, see [1] for the details. The result is as follows. String solution consists of a gauge field core of the radius $R_{g} \sim\left(1 / m_{G}\right) \ln \left(m_{G} / m_{L}\right)$, logarithmic tail $1 / m_{G} \lesssim r \lesssim 1 / m_{L}$ and exponential fall-off at larger $r$. Inside the core the profile functions are approximately constants $\left(\phi_{1} \sim 0, f \sim 1\right)$, while at the logarithmic tail we have

$$
\begin{aligned}
\phi_{1} & \approx \sqrt{\xi}\left(1-\frac{\ln \frac{1}{r m_{L}}}{\ln \frac{m_{G}}{m_{L}}}\right), \\
\phi_{2} & \approx \sqrt{\xi}, \\
f & \approx f_{W} \approx 0 .
\end{aligned}
$$

At the large $r \gg 1 / m_{L}$ fields exponentially approach their VEVs.

Calculating the energy on this configuration we arrive at the tension of the string

$$
T=\frac{2 \pi|\xi|}{\ln \frac{m_{G}}{m_{L}}}+\cdots
$$

where corrections are suppressed by powers of large logarithm $\log m_{G} / m_{L}$. The leading term here comes from quark kinetic energy $\left(\left(\phi_{1}^{\prime}\right)^{2}\right)$ integrated over intermediate region. Note, that the logarithmic suppression of the string tension is not specific for non-Abelian strings. Similar expression was found for the ANO string on a Higgs branch [26, 27]. The formula (3.5) should be contrasted with the exact result $T_{B P S}=2 \pi \xi$ for the BPS case (see, for example, [15]).

\subsection{Fermionic sector}

As was mentioned before, fermionic sector is essentially trivial. When the $\mu$ parameter is switched on, supersymmetry is broken in the orientational sector, and all the orientational modes are lifted. For small $\mu$ this can be derived by solving the Dirac equations in perturbation theory. It turns out that there is no zero mode, and the lowest mode has a mass

$$
m_{o r}=-\frac{\mu_{2} g_{2}^{2} \int_{0}^{\infty} \mathrm{d} y \frac{f_{N}^{2}(y) \phi_{1}^{2}(y)}{y \phi_{2}^{2}(y)}}{1-2 \int_{0}^{\infty} \mathrm{d} y \frac{f_{N}^{2}(y) \phi_{1}^{2}(y)}{y \phi_{2}^{2}(y)}} .
$$

In the orientational sector $\mathcal{N}=1$ supersymmetry survives which allows to generate the supertranslational modes from the bosonic fields. However the world sheet theory is trivial and therefore does not affect the string dynamics. 


\section{World sheet theory}

\subsection{Kinetic term}

Each particular solution (3.1) (say with $n^{l}=\delta^{l 1}$ ) breaks the $\mathrm{SU}(N)_{C+F}$ group down to $\mathrm{SU}(N-$ $1) \times \mathrm{U}(1)$, and therefore the $n^{P}$ span the moduli space

$$
\frac{\mathrm{SU}(N)_{C+F}}{\mathrm{SU}(N-1) \times U(1)}=\mathrm{CP}(N-1) .
$$

To derive a world sheet theory we promote translational and orientational moduli to slowly varying functions of the world sheet coordinates $t=x_{0}, z=x_{3}$. Namely, we will have translational moduli $x_{0}^{i}(t, z)$ (position of the string in the $\left(x_{1}, x_{2}\right)$ plane, $\left.i=1,2\right)$ and orientational moduli $n^{P}(t, z), P=1, \ldots, N$. They then become two-dimensional fields [10, 11], see [15] for a review. Translational sector is free and sterile, so we will study only the dynamics of the orientational modes. The gauge field acquires longitudinal components $[10,11,28]$

$$
A_{k}^{\mathrm{SU}(N)}=-i\left[\partial_{k} n \cdot \bar{n}-n \cdot \partial_{k} \bar{n}-2 n \cdot \bar{n}\left(\bar{n} \partial_{k} n\right)\right] \rho(r), \quad k=0,3,
$$

where $\rho$ is a profile function to be determined.

Substituting (3.1) and (4.2) into (2.8) we get $\mathrm{CP}(N-1)$ model

$$
S^{(1+1)}=2 \beta \int d t d z\left\{\left(\partial_{k} \bar{n} \partial_{k} n\right)+\left(\bar{n} \partial_{k} n\right)^{2}\right\}
$$

with the coupling constant $\beta$ given by [1]

$$
\beta \approx c \frac{2 \pi}{g_{2}^{2}} \frac{m_{G}^{2}}{m_{L}^{2}} \frac{1}{\ln ^{2} \frac{m_{G}}{m_{L}}} \sim \frac{|\mu|}{|m|} \frac{1}{\ln ^{2} \frac{g^{2}|\mu|}{|m|}} .
$$

This equation is in fact valid at a scale of the UV cutoff $m_{G} \sim \sqrt{\xi}$ of the world sheet theory. Then the running coupling constant of the $\mathrm{CP}(N-1)$ model is given by

$$
4 \pi \beta(\sqrt{\xi})=N \ln \frac{\sqrt{\xi}}{\Lambda_{C P}}
$$

where the scale $\Lambda_{C P}$

$$
\Lambda_{C P} \approx \sqrt{\xi} \exp \left\{- \text { const } \frac{|\mu|}{|m|} \frac{1}{\ln ^{2} \frac{g^{2}|\mu|}{|m|}}\right\} .
$$

This relation should be contrasted with $\mathcal{N}=2$ QCD string, for which the scale $\Lambda_{C P}$ was equal to scale $\Lambda_{\mathcal{N}=2}$ of the bulk QCD [15]. In the present case $\Lambda_{C P}$ is exponentially small, so the world sheet theory is weekly coupled in a wide region of energies $\gg \Lambda_{C P}$.

\subsection{World sheet potential}

In the case when the quark masses entering the Lagrangian (2.8) are non-identical, a potential on $n^{P}$ is generated. In the simplest case when all masses are real, this potential is given by [1]

$$
V_{1+1} \approx \frac{4 \pi|\mu|}{\ln \frac{m_{G}}{m_{L}}} \sum_{P=1}^{N} m_{P}\left|n^{P}\right|^{2} .
$$


The potential (4.7) has only one minimum and one maximum at generic $\Delta m_{A B}$. Other $(N-2)$ extreme points are saddle points. All these extreme points are located at

$$
n^{A}=\delta^{A A_{0}}, \quad A_{0}=1, \ldots, N
$$

and associated with the $Z_{N}$ strings. A value of the potential at a given extreme point coincides with the tension of the $A_{0}$-th $Z_{N}$ string,

$$
V_{1+1}\left(n^{A}=\delta^{A A_{0}}\right)=T_{A_{0}}, \quad A_{0}=1, \ldots, N .
$$

Absolute minimum (the unique vacuum) of (4.7) corresponds to the $Z_{N}$ string associated with winding of a squark with the smallest mass.

\subsection{Semilocal strings in the $\mathcal{N}=1$ limit}

In the case with "extra" quark flavors, $N_{f}>N_{c}$, the non-Abelian string of $\mathcal{N}=2$ QCD becomes semilocal and develops so-called size moduli $\rho_{K}, K=N_{c}+1, . ., N_{f}$ [23]. Consider the case when $\widetilde{N}=N_{f}-N_{c}=1$. The string in this case is BPS saturated, its tension given by

$$
T_{\text {semilocal }}=2 \pi \xi
$$

The world sheet theory for this string was derived in [23]:

$$
S_{S U S Y}^{2 d}=\int d^{2} x\left\{\left|\partial_{k}\left(\rho n_{P}\right)\right|^{2} \ln \frac{L}{|\rho|}+\frac{4 \pi}{g^{2}}\left[\left|\partial_{k} n_{P}\right|^{2}+\left(\bar{n}_{P} \partial_{k} n_{P}\right)^{2}\right]\right\},
$$

where $L$ is an IR cutoff.

Now consider $\mu$ deformation of our theory. We find that the string is no longer BPS. The scalar which develop VEV has smaller mass then the mass of the gauge boson. This corresponds to a type I superconductivity. The size $\rho$ is no longer a modulus cf. [32]. It develops a potential $\sim 1 /|\rho|^{2}$ in (4.11) which forces the semilocal string to shrink and become a local string.

\section{Physics of confined monopoles}

Above we discussed non-Abelian strings in $\mathcal{N}=2$ supersymmetric QCD deformed by a large mass term for the adjoint matter. In the limit of large $\mu$ this theory flows to $\mathcal{N}=1$ SQCD. The main question is, what happens to the confined monopoles as we pass on to the $\mathcal{N}=1$ QCD? In order to answer this question we derived and analyzed the effective theory on the string world sheet. It turns out that this theory is bosonic $\mathrm{CP}(N-1)$ model (4.3) with shallow potential (4.7) generated by small quark mass differences. The fermionic superpartners of the bosonic orientational moduli $n^{l}$ present in the $\mathcal{N}=2$ limit become heavy at large $\mu$ and decouple.

Since our four-dimensional theory is in the Higgs phase for squarks, 't Hooft-Polyakov monopoles present in the theory in the $\mathcal{N}=2$ limit of small $\mu$ are confined by non-Abelian strings and serve as junctions of two distinct strings [11, 12,29]. In the effective world sheet theory on the non-Abelian string they are seen as kinks interpolating between different vacua of $\mathrm{CP}(N-1)$ model, see [15] for a review. 
In the large $\mu$ limit adjoint fields decouple. Therefore we could expect quasiclassically that the confined monopoles disappear in this limit. This indeed happen for non-equal quark masses. If quark mass differences are non-zero, a potential (4.7) is generated. It does not have multiple local minima, therefore kinks (confined monopoles of the bulk theory) become unstable and disappear.

However, in the equal quark mass case, the analysis of the quantum world sheet theory shows that confined monopoles do survive. The monopoles are represented by kinks in the effective $\mathrm{CP}(N-1)$ model on the non-Abelian string. The $\mathrm{CP}(N-1)$ model is at strong coupling. It was solved by Witten [30] in the large $N$ approximation. It was shown that kinks in this model are in a confinement phase. In terms more suitable for application to monopole physics of the bulk theory this can be understood as follows, see also [15] for a more detail review.

The vacuum structure of the $C P(N-1)$ model was studied in [31]. It was shown that there is a unique vacuum. There are, however, of order $N$ quasi-vacua, which become stable in the limit $N \rightarrow \infty$, since an energy split between the neighboring quasi-vacua is $O(1 / N)$. Thus, one can imagine a kink interpolating between the true vacuum and the first quasi-vacuum and the anti-kink returning to the true vacuum. Linear confining potential between kink and anti-kink is associated with excited quasi-vacuum.

This two dimensional confinement of kinks was interpreted in terms of strings and monopoles of the bulk theory in [28]. The fine structure of vacua in $\operatorname{CP}(N-1)$ model on the non-Abelian string means that $N$ elementary strings are split by quantum effects and have slightly different tensions. The difference between the tensions of "neighboring" strings is proportional to $\Lambda_{C P}^{2}$, see (4.6). Therefore monopoles, in addition to the four dimensional confinement (which ensures that they are attached to the string), acquire two-dimensional confinement along the string. Monopole and antimonopole connected by a string with larger tension form a mesonic bound state.

\section{Acknowledgments}

The authors are grateful to Mikhail Shifman for very useful and stimulating discussions. The work of A. Y. was supported by William I. Fine Theoretical Physics Institute of the University of Minnesota and by RFBR Grant No. 18-02-00048. The work of E. I. was funded by RFBR Grants No. 18-32-00015 and No. 18-02-00048.

\section{References}

[1] E. Ievlev and A. Yung, Phys. Rev. D 95, 125004 (2017) [arXiv:1704.03047].

[2] Y. Nambu, Phys. Rev. D 10, 4262 (1974);

G. 't Hooft, Proc. of the E.P.S. Int. Conf. on High Energy Physics, Palermo, 23-28 June, 1975 ed. A. Zichichi (Editrice Compositori, Bologna, 1976); Nucl. Phys. B 190, 455 (1981); S. Mandelstam, Phys. Rept. 23, 245 (1976).

[3] N. Seiberg and E. Witten, Nucl. Phys. B426, 19 (1994), (E) B430, 485 (1994) [hepth/9407087].

[4] N. Seiberg and E. Witten, Nucl. Phys. B431, 484 (1994) [hep-th/9408099].

[5] M. R. Douglas and S. H. Shenker, Nucl. Phys. B 447, 271 (1995) [hep-th/9503163]. 
[6] A. Hanany, M. J. Strassler and A. Zaffaroni, Nucl. Phys. B 513, 87 (1998) [hepth/9707244].

[7] M. Strassler, Prog. Theor. Phys. Suppl. 131, 439 (1998) [hep-lat/9803009].

[8] A. I. Vainshtein and A. Yung, Nucl. Phys. B 614, 3 (2001) [arXiv:hep-th/0012250].

[9] A. Hanany and D. Tong, JHEP 0307, 037 (2003) [arXiv:hep-th/0306150].

[10] R. Auzzi, S. Bolognesi, J. Evslin, K. Konishi and A. Yung, Nucl. Phys. B 673, 187 (2003) [arXiv:hep-th/0307287].

[11] M. Shifman and A. Yung, Phys. Rev. D 70, 045004 (2004) [arXiv:hep-th/0403149].

[12] A. Hanany and D. Tong, JHEP 0404, 066 (2004) [arXiv:hep-th/0403158].

[13] D. Tong, hep-th/0509216.

[14] M. Eto, Y. Isozumi, M. Nitta, K. Ohashi and N. Sakai, J. Phys. A 39, R315 (2006) [arXiv:hep-th/0602170].

[15] M. Shifman and A. Yung, Rev. Mod. Phys. 791139 (2007) [arXiv:hep-th/0703267]; an expanded version in Cambridge University Press, (2009).

[16] D. Tong, Annals Phys. 324, 30 (2009) [arXiv:0809.5060 [arXiv:hep-th]].

[17] M. Shifman and A. Yung, Phys. Rev. D 72, 085017 (2005) [arXiv:hep-th/0501211].

[18] M. Edalati and D. Tong, JHEP 0705, 005 (2007) [arXiv:hep-th/0703045].

[19] D. Tong, JHEP 0709, 022 (2007) [arXiv:hep-th/0703235].

[20] M. Shifman and A. Yung, Phys. Rev. D 77, 125016 (2008) Erratum: [Phys. Rev. D 79, 049901 (2009)] [arXiv:0803.0158].

[21] M. Shifman and A. Yung, Phys. Rev. D 77, 125017 (2008) Erratum: [Phys. Rev. D 81, 089906 (2010)] [arXiv:0803.0698].

[22] P. A. Bolokhov, M. Shifman and A. Yung, Phys. Rev. D 79, 085015 (2009) [arXiv:0901.4603].

[23] M. Shifman, W. Vinci and A. Yung, Phys. Rev. D 83, 125017 (2011) [arXiv:1104.2077].

[24] M. Shifman and A. Yung, Int. J. Mod. Phys. A 29, no. 27, 1430064 (2014) [arXiv:1410.2900 [hep-th]].

[25] A. A. Penin, V. A. Rubakov, P. G. Tinyakov and S. V. Troitsky, Phys. Lett. B 389, 13 (1996) [hep-ph/9609257].

[26] A. Yung, Vortices on the Higgs Branch of the Seiberg-Witten Theory, Nucl. Phys. B 562, 191 (1999) [hep-th/9906243].

[27] K. Evlampiev and A. Yung, Flux Tubes on Higgs Branches in SUSY Gauge Theories, Nucl. Phys. B 662, 120 (2003) [hep-th/0303047].

[28] A. Gorsky, M. Shifman and A. Yung, Non-Abelian Meissner effect in Yang-Mills theories at weak coupling, Phys. Rev. D 71, 045010 (2005) [hep-th/0412082].

[29] D. Tong, Monopoles in the Higgs phase, Phys. Rev. D 69, 065003 (2004), [hepth/0307302].

[30] E. Witten, Instantons, the Quark Model, and the 1/n Expansion, Nucl. Phys. B 149, 285 (1979).

[31] E. Witten, Theta Dependence in the Large N Limit of Four-Dimensional Gauge Theories, Phys. Rev. Lett. 81, 2862 (1998), [hep-th/9807109].

[32] A. Achucarro and T. Vachaspati, Semilocal and electroweak strings, Phys. Rept. 327, 347 (2000). 